\title{
A Multiobjective Multi-Item Inventory Control Problem in Fuzzy-Rough Environment Using Soft Computing Techniques
}

\author{
Dipak Kumar Jana, ${ }^{1}$ K. Maity, ${ }^{2}$ M. Maiti, ${ }^{3}$ and T. K. Roy ${ }^{4}$ \\ ${ }^{1}$ Department of Engineering Science, Haldia Institute of Technology, Haldia, West Bengal 721657, India \\ ${ }^{2}$ Department of Mathematics, Mugberia Gangadhar Mahavidyalaya, Mugberia, Purba Medinipur, West Bengal 721425, India \\ ${ }^{3}$ Department of Applied Mathematics with Oceanology and Computer Programming, Vidyasagar University, Medinipur, \\ West Bengal 721 102, India \\ ${ }^{4}$ Department of Mathematics, Bengal Engineering and Science University, Shibpur, Howrah, West Bengal 711103, India
}

Correspondence should be addressed to Dipak Kumar Jana; dipakjana@gmail.com

Received 14 October 2013; Revised 19 January 2014; Accepted 22 January 2014; Published 7 April 2014

Academic Editor: Henry Schellhorn

Copyright (c) 2014 Dipak Kumar Jana et al. This is an open access article distributed under the Creative Commons Attribution License, which permits unrestricted use, distribution, and reproduction in any medium, provided the original work is properly cited.

\begin{abstract}
The optimal production and advertising policies for an inventory control system of multi-item multiobjective problem under a single management are formulated as an optimal control problem with resource constraints under inflation and discounting in fuzzy rough (Fu-Ro) environment. The objectives and constraints in Fu-Ro are made deterministic using fuzzy rough expected values method (EVM). Here, the production and advertisement rates are unknown and considered as control (decision) variables. The production, advertisement, and demand rates are functions of time $t$. Maximization of the total proceed from perfect and imperfect units and minimization of the total cost consisting of production, holding, and advertisement costs are formulated as optimal control problems and solved directly using multiobjective genetic algorithm (MOGA). In another method for solution, membership functions of the objectives are derived and the multi-objective problems are transformed to a single objective by the convex combination of the membership functions and then the problem is solved by generalized reduced gradient (GRG) method. Finally, numerical experiment and graphical representation are provided to illustrate the system.
\end{abstract}

\section{Introduction}

From financial standpoint, an inventory represents a capital investment and a lot of researchers' works have been done since the Second World War. Most of the classical inventory models did not take into account the effects of inflation and time value of money. This has happened mostly because of the belief that inflation and time value of money will not influence the cost and price components (i.e., the inventory policy) to any significant degree. But, during last few decades, due to high inflation and consequent sharp decline in the purchasing power of money in the developing countries like Brazil, Argentina, India, Bangladesh, and so forth, the financial situation has been changed and so it is not possible to ignore the effect of inflation and time value of money any further. Following Buzacott [1], Misra [2] extended his approaches to different inventory models with finite replenishment, shortages, and so forth, by considering the time value of money, different inflation rates for the costs. Also Lo et al. [3] developed an integrated productioninventory model with a varying rate of deterioration under imperfect production process, partial backordering, and inflation. Again, some researchers (cf. Cho [4] and others) have assumed depreciation rate of sales as a function of time, $t$. This assumption is supported by a general fact that, as time goes on, a firm usually faces more competition (thus it may lose its sales at an increasing rate). Again, to boost up the sale, the management goes for advertisement and thus advertisement policy plays an important role of increasing the demand. Also, a promotional cost (cf. Datta et al. [5], etc.) is introduced to provide the advertisement that increases the demand. Sivashankari and Panayappan [6] have developed a production inventory model with reworking of imperfect production, scrap, and shortages. Krishnamoorthi 
and Panayappan [7] have implemented an EPQ model for imperfect production systems with rework and incorporating a multidelivery policy. Mandal and Khan [8] have developed the theory and methodology for the JELS model in onevendor, multicustomer situations when costs are fuzzy and lot sizes are deterministic.

Uncertainty, such as randomness, fuzziness, and/or roughness, is common in real-life problems. In 1965, Zadeh [9] first has introduced the idea of fuzzy set theory. The fuzzy set theory has been well developed and applied in a wide variety of real problems. Possibility theory was also proposed by Zadeh [10] and developed by many researchers such as Dubois and Prade [11] and Jana et al. [12]. However, in a decision-making process, we may face a hybrid uncertain environment where fuzziness and roughness exist at the same time. In such cases, a fuzzy rough variable is a useful tool. Fuzziness and roughness play an important role for the uncertainty problems. The concept of fuzzy rough sets introduced by Dubois and Prade [13] plays a key role in dealing with the two types of uncertainty simultaneously.

Dubois and Prade [14] first introduced the fuzzification problem of rough sets. Fuzzy programming and rough programming have been proposed for decisions under uncertainty environment. In these programming models, roughness, and fuzziness are considered as separate aspects. Several researchers have considered the issue of combining fuzziness and roughness in a general framework for the study of fuzzy rough sets. Morsi and Yakout [15] have developed an approximation for fuzzy rough sets. At present, the fuzzy rough set has been applied to practical problems. Furthermore, the fuzzy rough set theory was used to extract fuzzy decision rules from fuzzy information systems. In 2008, Xu and Zhao [16] also developed a class of fuzzy rough expected value multiobjective decision-making models and its application to inventory problems. However, no attempt has been made that includes all selling prices, production cost, holding cost, and advertisement cost as fuzzy rough variables. It has been considered within the multiobjective inventory decisionmaking system with defective items. Therefore, there is a strong motivation for further research in this area. Based on the concept of fuzzy rough variables, we develop a fuzzy rough model applicable to multiobjective multi-item production inventory problems.

The multiobjective decision-making (MODM) problems have been formulated and solved in many areas like production inventory control problem, air pollution, structural analysis, and transportation (cf. Tao and $\mathrm{Xu}$ [17], etc.). Till now, only a few papers on MODM have been published in the field of inventory control system in fuzzy-rough environment. Maity and Maiti [18] formulated production inventory control problem in fuzzy environment. $\mathrm{Xu}$ and Zhao [19] have developed a multiobjective decision-making model with fuzzy rough coefficients and made its application to the inventory problem.

Genetic algorithm approach was first proposed by Holland [20]. Because of its generality, it has been successfully applied to many optimization problems for its several advantages over conventional optimization methods. There are several approaches using genetic algorithms to deal with the multiobjective optimization problems. These algorithms can be classified into two types-(i) nonelitist MOGA and (ii) elitist MOGA. Among nonelitist MOGAs, Fonseca and Fleming's MOGA [21] and Srinivas and Deb's NSGA [22] enjoyed more attention. Among nonelitist MOGAs, Srinivas and Deb's NSGA [22] is discussed here and used to solve a multiobjective inventory model. A fast and elitist MOGA is developed following Deb et al. [23], Jana et al. [24], and others and used to solve the present models also. This algorithm is named Fast and Elitist Multiobjective Genetic Algorithm (FEMOGA).

For the inventory problem, the classical inventory decision-making models are normally considered for a single-item. However, single-item inventories seldom occur, whereas multi-item inventories are common in real-life situations. Several researchers (cf. Lee and Yao [25], Taleizadeh et al. [26], and Balkhi and Foul [27]) discussed multiitem classical inventory models under resource constraints. In reality, the parameters involved in solving inventory problems may be uncertain and fuzzy rough in nature. For example, production costs of an item may be dependent upon the total quantity to be produced. But in the inventory system the amount produced within a scheduling period may be uncertain and may range within an interval arising from specific requirements, such as local conditions and customer demand. In such situations, the fuzzy rough theory can be used for the formulation of inventory models. Therefore, there is a strong motivation for the present research in the area, where we consider fuzzy rough constrained multi-item inventory problems.

In this paper, advertising and production policies are developed for a multi-item multiobjective production inventory control problem. The system is under the control of inflation and discounting. We sell the defective items at production center by reduced price. The salvage price for selling the marginal stock is also included. The warehouse to store the items is of limited capacity and the investment is also limited; these are fuzzy rough in nature. The relevant inventory costs like production, holding, and advertisement costs are considered as fuzzy rough variables. Here, we consider multiobjective production inventory control problems of which the first objective is the total profit which is maximized and the second is the minimization of the total cost. This multiobjective problem is formulated as an optimal control problem and solved numerically using MOGA and GRG techniques (cf. Gabriele and Ragsdell [28]). Optimum productions, the stock levels, and demands are determined with different types of advertising policies for different items. The model is illustrated through numerical example.

\section{Mathematical Preliminaries}

2.1. Fuzzy Rough Set. In this section, we present some basic knowledge on fuzzy rough set theory. These results are crucial for the rest of this paper and interested readers may consult [19]. The fundamental concept in rough set theory is the approximation space. Suppose $U \neq \Phi$ is a finite set of objectives. Let $R \in U \times U$ be an equivalence relation on $U$; 
that is, $R$ is reflexive, symmetric, and transitive. Then, the pair $(U, R)$ is called an approximation space.

The equivalence relation $R$ forms a partition of $U$; that is, the families $\left\{X_{1}, X_{2}, \ldots, X_{m}\right\}$ satisfy $X_{i} \subseteq U, X_{i} \neq \phi$, $X_{i} \cap X_{j}=\phi(i \neq j)$, and $\bigcup X_{i}=U$. Conversely, $C=$ $\left\{X_{1}, X_{2}, \ldots, X_{m}\right\}$, which is a partition of $U$, determines an equivalence relation on $U$ in such way that $(x, y) \in R$ if $x, y \in X_{i}, i=1,2, \ldots, m$. We use $U \mid R$ to denote the set of all equivalence classes of $R$ and use $[x]_{R}$ to denote an equivalence class in $R$ containing an element $x \in U$. Then, $U \mid R=\left\{[x]_{R} \mid x \in U\right\}$.

Definition 1. Let $U$ be a universe and $X$ a set representing a concept. Then, its lower approximation is defined by (cf. Xu and Zhou [29], Jana et al. [30])

$$
\underline{X}=\left\{x \in U \mid R^{-1}(x) \subset X\right\}
$$

and the upper approximation is defined by

$$
\bar{X}=\bigcup_{x \in X} R(x) .
$$

Definition 2. The collection of all sets having the same lower and upper approximations is called a rough set, denoted by $(\underline{X}, \bar{X})$. The figure of a rough set is depicted in Figure 1 .

Example 3. Let $\xi$ focus on the continuous set in the onedimension real space $R$. There are still some vague sets which cannot be directly fixed and need to be described by the rough approximation. For example, let $R$ be the universe, a similarity relation is defined as $a \simeq b$ if and only if $|a-b| \leq 10$. We have that, for the set $[20,50]$, its lower approximation $\underline{[20,50]}=[30,40]$ and its upper approximation $\overline{[20,50]}=$ $[10,60]$. Then, the upper and lower approximation of the set $[20,50]$ make up a rough set $([30,40],[10,60])$ which is the collection of all sets having the same lower approximation $[30,40]$ and upper approximation $[10,60]$.

\subsection{Fuzzy Rough Variables}

Definition 4. A fuzzy rough variable $\xi$ is a fuzzy variable with uncertain parameter $\rho \in X$, where $X$ is approximated by $(\underline{X}, \bar{X})$ according to the similarity relation $R$, namely, $\underline{X} \subseteq$ $X \subseteq \bar{X}$.

For convenience, we usually denote $\rho \vdash(\underline{X}, \bar{X})_{R}$ expressing that $\rho$ is in some set $A$ which is approximated by $(\underline{X}, \bar{X})$

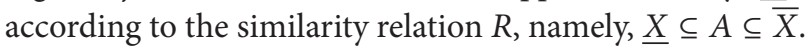

Example 5. We consider the LR fuzzy variable $\xi$ with the following membership function:

$$
\mu_{\xi}(x)= \begin{cases}L\left(\frac{\rho-x}{\alpha}\right), & \text { if } \rho-\alpha<x<\rho, \\ 1, & \text { if } x=\rho, \\ L\left(\frac{x-\rho}{\beta}\right), & \text { if } \rho<x<\rho+\beta,\end{cases}
$$

where $\rho \vdash([1,2],[0,3])_{R}$; then, $\xi$ is a fuzzy rough variable.
Example 6. Let $U=[-5,5]$ be a universe and let $R$ be an equivalent relation on $U$ defined by the partition $\{[-5,-4),[-4,-3),[-3,-2),[-2,-1),[-1,0),[0,1),[1,2),[2$, $3),[3,4),[4,5]\}$, and $X=[-3.5,-1.2][2.1,4.3]$. Denote $X^{\alpha}=\left\{x \mid \mu_{X}^{R}(x) \geq \alpha\right\}(0<\alpha<1)$. It follows from the concept of rough membership that

$$
X^{\alpha}= \begin{cases}{[-4 ;-1][2 ; 5] ;} & \text { if } 0<\alpha<0.3 \\ {[-4 ;-1][2 ; 4] ;} & \text { if } 0.3<\alpha<0.5 \\ {[-3 ;-2][2 ; 4] ;} & \text { if } 0.5<\alpha<0.8 \\ {[-3 ;-2][2 ; 4] ;} & \text { if } 0.8<\alpha<0.9 \\ {[-3 ;-2][3 ; 4] ;} & \text { if } 0.9<\alpha<1\end{cases}
$$

2.2.1. Equivalent Crisp Model for Fu-Ro EVM. For the multiobjective model (5) with Fu-Ro parameters, we cannot deal with it directly and should use some tools to make it have mathematical meaning; then, we can solve it. According to $\mathrm{Xu}$ and Zhou [29], we employ the expected value operator to transform the fuzzy rough model into Fu-Ro EVM. Consider the following multiobjective decision-making model (5) with fuzzy rough coefficients:

$$
\begin{array}{ll}
\max & f_{1}(x, \xi), f_{2}, \ldots, f_{m}(x, \xi) \\
\text { s.t. } & g_{r}(x, \xi) \leq 0, \quad r=1,2, \ldots, p \\
& x \in X,
\end{array}
$$

where $x$ is a $n$-dimensional decision vector, $\xi=$ $\left(\xi_{1}, \xi_{2}, \xi_{3}, \ldots, \xi_{n}\right)$ is a Fu-Ro vector, and $f_{i}(x, \xi)$ are objective functions, $i=1,2, \ldots, m$. Because of the existence of $\mathrm{Fu}$-Ro vector, problem (5) is not well-defined. That is, the meaning of maximizing $f_{i}(x, \xi), i=1,2, \ldots, m$ is not clear and constraints $g_{r}(x, \xi), 0, r=1,2, \ldots, p$ do not define a deterministic feasible set. In the following, we use Fu-Ro EVM to deal with the meaningless model.

2.3. Fu-Ro EVM. Based on the definition of the expected value of fuzzy rough events $f_{i}$ and $g_{r}$, the general model for $\mathrm{Fu}-\mathrm{Ro}$ EVM is proposed as follows:

$$
\begin{array}{ll}
\max & E\left[f_{1}(x, \xi), f_{2}(x, \xi), \ldots, f_{m}(x, \xi)\right] \\
\text { s.t. } & E\left[g_{r}(x, \xi)\right] \leq 0, \quad r=1,2, \ldots, p \\
& x \in X .
\end{array}
$$

Definition 7. If $x^{*}$ is an efficient solution of problem (6), one call it as a fuzzy rough expected efficient solution. Clearly, the problem (6) is a multiobjective with crisp parameters. Then, one can convert it into a single-objective programming by traditional method of weight sum. Consider

$$
\begin{array}{ll}
\max & \omega_{i} \sum_{i=1}^{n} E\left[f_{i}(x, \xi)\right] \\
\text { s.t. } & E\left[g_{r}(x, \xi)\right] \leq 0, \quad r=1,2, \ldots, p \\
& x \in X .
\end{array}
$$




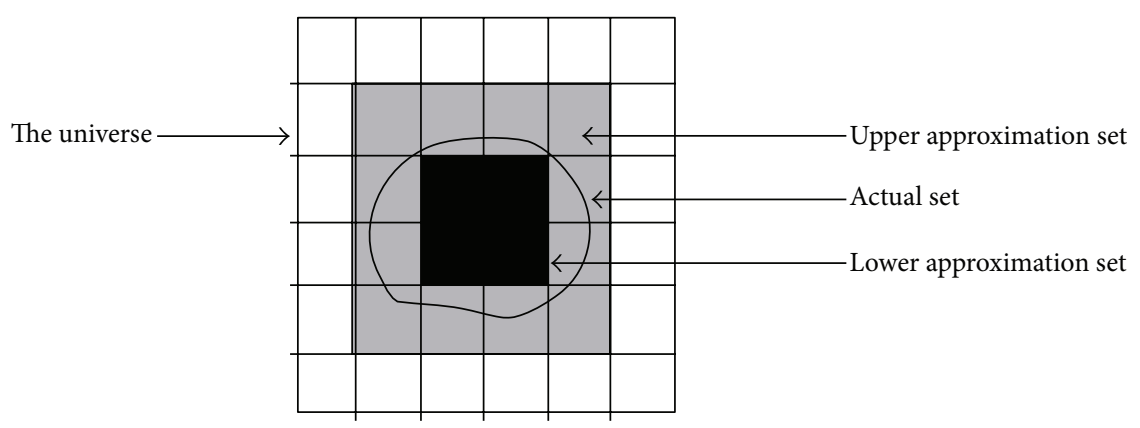

Figure 1: A rough set.

Lemma 8. Assume that $\xi$ and $\eta$ are the introduction of variables with finite expected values. Then, for any real numbers a and $b$, one has

$$
E[a \xi+b \eta]=a E[\xi]+b E[\eta] .
$$

Proof. The proof of the Lemma is in [29].

Theorem 9. If trapezoidal fuzzy rough numbers $\tilde{\bar{c}}_{i j}$ are defined as $\tilde{\bar{c}}_{i j}(\lambda)=\left(\bar{c}_{i j 1}, \bar{c}_{i j 2}, \bar{c}_{i j 3}, \bar{c}_{i j 4}\right)$ with $\bar{c}_{i j t} \quad \vdash$ ([c $\left.\left.c_{i j t 2}, c_{i j t 3}\right],\left[c_{i j t 1}, c_{i j t 4}\right]\right)$, for $i=1,2, \ldots, m, j=1,2, \ldots, n$, $t=1,2,3,4,0 \leq c_{i j t 1} \leq c_{i j t 2}<c_{i j t 3} \leq c_{i j t 4}$, then $E\left[\widetilde{\bar{c}}_{1}^{T}\right], E\left[\tilde{\bar{c}}_{2}^{T}\right], \ldots, E\left[\tilde{\bar{c}}_{n}^{T}\right]$ is equivalent to

$$
\frac{1}{16} \sum_{j=1}^{n} \sum_{t=1}^{4} \sum_{k=1}^{4} c_{1 j t k}, \frac{1}{16} \sum_{j=1}^{n} \sum_{t=1}^{4} \sum_{k=1}^{4} c_{2 j t k}, \ldots, \frac{1}{16} \sum_{j=1}^{n} \sum_{t=1}^{4} \sum_{k=1}^{4} c_{n j t k} .
$$

Proof. The proof of the theorem is in [29].

Theorem 10. If trapezoidal fuzzy rough numbers $\tilde{\bar{a}}_{r j}$ and $\tilde{\bar{b}}_{r}$ are defined as follows:

$$
\begin{aligned}
& \tilde{\bar{a}}_{r j}(\lambda)=\left(\bar{a}_{r j 1}, \bar{a}_{r j 2}, \bar{a}_{r j 3}, \bar{a}_{r j 4}\right) \text { with } \bar{a}_{r j t} \vdash\left(\left[a_{r j t 2}, a_{r j t 3}\right],\right. \\
& \left.\left[a_{r j t 1}, a_{r j t 4}\right]\right), \\
& \tilde{\bar{b}}_{r}(\lambda)=\left(\bar{b}_{r 1}, \bar{b}_{r 2}, \bar{b}_{r 3}, \bar{b}_{r 4}\right) \text { with } \bar{b}_{r t} \vdash\left(\left[b_{r t 2}, b_{r t 3}\right],\right. \\
& \left.\left[b_{r t 1}, b_{r t 4}\right]\right),
\end{aligned}
$$

for $r=1,2, \ldots, p, t=1,2,3,4,0 \leq a_{r t 1} \leq a_{r t 2}<a_{r t 3} \leq$ $a_{r t 4}, 0 \leq b_{r t 1} \leq b_{r t 2}<b_{r t 3} \leq b_{r t 4}$, then $E\left[\tilde{\bar{a}}_{r j}^{T}\right] \leq E\left[\tilde{\bar{b}}_{r j}\right], r=$ $1,2, \ldots, p$ is equivalent to

$$
\frac{1}{16} \sum_{j=1}^{n} \sum_{t=1}^{4} \sum_{k=1}^{4} a_{r j t k} \leq \frac{1}{16} \sum_{t=1}^{4} \sum_{k=1}^{4} b_{r t k}, \quad r=1,2, \ldots, p .
$$

Proof. The proof of the theorem is in [29].

2.4. Multiobjective Genetic Algorithm (MOGA). Fast and Elitist Multiobjective Genetic Algorithm has the following two important components.

(a) Division of a population of solutions into subsets having nondominated solutions: consider a problem having $M$ objectives and take a population $P$ of feasible solutions of the problem of size $N$. We like to partition $P$ into subsets $F_{1}, F_{2}, \ldots, F_{k}$, such that every subset contains nondominated solutions, but every solution of $F_{i}$ is not dominated by any solution of $F_{i+1}$, for $i=1,2, \ldots, k-1$. To do this for each solution, $x$, of $P$, calculate the following two entities.

(i) Number of solutions of $P$ which dominate $x$. Let it be $n_{x}$.

(ii) Set of solutions of $P$ that are dominated by $x$. Let it be $S_{x}$.

The above two steps require $O\left(M N^{2}\right)$ computations. Clearly $F_{1}$ contains every solution $x$ having $n_{x}=0$. Now for each solution $x \in F_{1}$, visit every member $y$ of $S_{x}$ and decrease $n_{y}$ by 1 . In doing so if, for any member $y, n_{y}=0$, then $y \in F_{2}$. In this way $F_{2}$ is constructed. The above process is continued to every member of $F_{2}$ and thus $F_{3}$ is obtained. This process is continued until all subsets are identified. For each solution $x$ in the second or higher level of nondominated subsets, $n_{x}$ can be at most $N-1$. So each solution $x$ will be visited at most $N-1$ times before $n_{x}$ becomes zero. At this point, the solution is assigned a subset and will never be visited again. Since there is at most $N-1$ such solutions, the total complexity is $O\left(N^{2}\right)$. So overall complexity of this component is $O\left(M N^{2}\right)$.

(b) Determine distance of a solution from other solutions of a subset: to determine distance of a solution from other solutions of a subset the following steps are followed.

(i) First sort the subset according to each objective function values in ascending order of magnitude.

(ii) For each objective function, the boundary solutions are assigned an infinite distance value (a large value).

(iii) All other intermediate solutions are assigned a distance value for the objective, equal to the absolute normalized difference in the objective values of two adjacent solutions. 
(iv) This calculation is continued with other objective functions.

(v) The overall distance of a solution from others is calculated as the sum of individual distance values corresponding to each objective.

Since $M$ independent sorting of at most $N$ solutions (in case the subset contains all the solutions of the population) is involved, the above algorithm has $O(M N \log N)$ computational complexity.

Using the above two operations, proposed multiobjective genetic algorithm takes the following form.

(1) Set probability of crossover $p_{c}$ and probability of mutation $p_{m}$.

(2) Set iteration counter $T=1$.

(3) Generate initial population set of solution $P(T)$ of size $N$.

(4) Select solution from $P(T)$ for crossover and mutation.

(5) Made crossover and mutation on selected solution and get the child set $C(T)$.

(6) Set $P_{1}=P(T) U C(T) / /$, where $U$ stands for union operation.

(7) Divide $P_{1}$ into disjoint subsets having nondominated solutions. Let these sets be $F_{1}, F_{2}, \ldots, F_{k}$.

(8) Select maximum integer $n$ such that order of $P_{2}\left(=F_{1} U F_{2} U \cdots U F_{n}\right) \leq N$.

(9) If $O\left(P_{2}\right)<N$ sort solutions of $F_{n+1}$ in descending order of their distance from other solutions of the subset, then select first $N-O\left(P_{2}\right)$ solutions from $F_{n+1}$ and add with $P_{2}$, where $O\left(P_{2}\right)$ represents order of $P_{2}$.

(10) Set $T=T+1$ and $P(T)=P_{2}$.

(11) If termination condition does not hold, go to Step 4.

(12) Output: $P(T)$

(13) End algorithm.

MOGAs that use nondominated sorting and sharing are mainly criticized for their

(i) $\mathrm{O}\left(M N^{3}\right)$ computational complexity;

(ii) nonelitism approach;

(iii) the need for specifying a sharing parameter to maintain diversity of solutions in the population.

In the above algorithm, these drawbacks are overcome. Since in the above algorithm computational complexity of Step 7 is $O\left(M N^{2}\right)$, Step 9 is $O(M N \log N)$, and other steps are $\leq O(N)$, so overall time complexity of the algorithm is $\mathrm{O}\left(M N^{2}\right)$. Here, selection of new population after crossover and mutation on old population is done by creating a mating pool by combining the parent and offspring population and among them, best $N$ solutions are taken as solutions of new population. By this way, elitism is introduced in the algorithm. When some solutions from a nondominated set
$F_{j}$ (i.e., a subset of $F_{j}$ ) are selected for new population, those are accepted whose distance compared to others (which are not selected) are rejected; that is, isolated solutions are accepted. In this way, taking some isolated solutions in the new population, diversity among the solutions is introduced in the algorithm, without using any sharing function. Since computational complexity of this algorithm $<O\left(M N^{3}\right)$ and elitism are introduced, this algorithm is named FEMOGA. Time complexity of NSGA can be reduced to $O\left(M N^{2}\right)$ if Step 4 of NSGA is done following Step 7 of above FEMOGA, but the deeded for sharing function in NSGA cannot be removed. Different procedures of the above FEMOGA are discussed in the following section. Procedures for NSGA can easily be developed similarly.

\section{Procedures of the Proposed FEMOGA}

(a) Representation: a " $K$ dimensional real vector" $X=$ $\left(x_{1}, x_{2}, \ldots, x_{K}\right)$ is used to represent a solution, where $x_{1}, x_{2}, \ldots, x_{K}$ represent different decision variables of the problem such that constraints of the problem are satisfied.

(b) Initialization: $N$ solutions $X_{1}, X_{2}, X_{3}, \ldots, X_{N}$ are randomly generated by random number generator from the search space such that each $X_{i}$ satisfies the constraints of the problem. This solution set is taken as initial population $P(1)$. Also set $p_{c}=0.3, p_{m}=0.2$, and $T=1$.

(c) Crossover:

(i) selection for crossover: for each solution of $P(T)$ generate a random number $r$ from the range $[0 \cdots 1]$. If $r<p_{c}$, then the solution is taken for crossover;

(ii) crossover process: crossover takes place on the selected solutions. For each pair of coupled solutions $Y_{1}, Y_{2}$, a random number $c$ is generated from the range $[0 \cdots 1]$ and offsprings $Y_{11}$ and $Y_{21}$ are calculated by $Y_{11}=c Y_{1}+(1-c) Y_{2}$, $Y_{21}=c Y_{2}+(1-c) Y_{1}$.

(d) Mutation:

(i) selection for mutation: for each solution of $P(T)$ generate a random number $r$ from the range $[0 \cdots 1]$. If $r<p_{m}$, then the solution is taken for mutation;

(ii) mutation process: to mutate a solution $X=$ $\left(x_{1}, x_{2}, x_{3}, \ldots, x_{K}\right)$ select a random integer $r$ in the range $[1 \cdots k]$. Then replace $x_{r}$ by randomly generated value within the boundary of $r$ th component of $X$.

(e) Division of $P(T)$ into disjoint subsets having nondominated solutions: following the discussions of the previous section Algorithm 1 is developed for this purpose.

(f) Determine distance of a solution of subset $F$ from other solutions: Algorithm 2 is used for this purpose. 


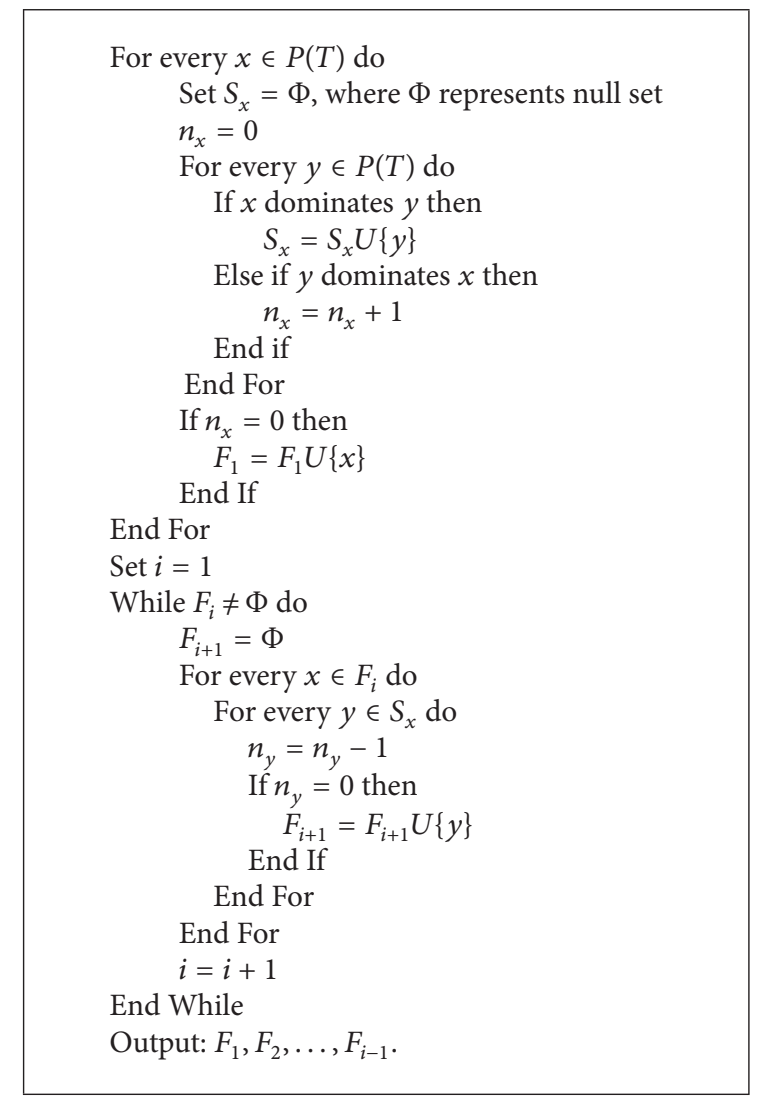

Algorithm 1

Set $n=$ number of solutions in $F$

For every $x \in F$ do

$x_{\text {distance }}=0$

End For

For every objective $m$ do

Sort $F$, in ascending order of magnitude of $m$ th objective.

$F[1]=F[n]=M$, where $M$ is a big quantity.

For $i=2$ to $n-1$ do End For$$
F[i]_{\text {distance }}=F[i]_{\text {distance }}+(F[i+1] \cdot \text { objm }-F[i-1] \cdot \text { objm }) /\left(f_{m}^{\max }-f_{m}^{\min }\right)
$$

End For

In the algorithm $F[i]$ represents $i$ th solution of $F$, $F[i]$. objm represent $m$ th objective value of $F[i] . f_{m}^{\max }$ and $f_{m}^{\min }$ represent the maximum and minimum values of $m$ th objective function.

\section{Production-Inventory Model in Fuzzy-Rough Environment}

\subsection{Notations}

$n$ : number of items,

$T$ : time length of the cycle,

Algorithm 2

$\widetilde{\bar{M}}$ : fuzzy rough maximum space available for storage,

$\widetilde{\bar{Z}}$ : fuzzy rough maximum investment costs.

For ith Item

$D_{i}(t)$ : rate of demand at time $t$,

$U_{i}(t)$ : production rate at time $t=u_{i 0}-u_{i 1} e^{-u_{i 2} t}$, where $u_{i 0}$, $u_{i 1}$, and $u_{i 2}$ are the control (decision) variables,

$X_{i}(t)$ : the inventory level at time $t$,

$\beta_{i}$ : rate of depreciation, 
$\delta_{i}$ : imperfect production rate,

$a_{i}$ : storage area for per unit item,

$V_{i}(t): v_{i 0} e^{-\gamma_{i} t}=$ the rate of demand created by the advertisement at time $t$ where $V_{i}(t)$ is the control (decision) variable and $v_{i 0}, \gamma_{i}$ are positive constants,

$b_{i}$ : salvage value which is also called reduction selling price for marginal stock,

$b_{\delta i}$ : reduced selling price for defective item,

$r_{i}$ : discount rate,

$k_{i}$ : rate of inflation,

$R_{i}$ : net discount rate of inflation $=r_{i}-k_{i}$

$\widetilde{\bar{C}}_{u i}$ : fuzzy rough production cost per unit item,

$\widetilde{\bar{C}}_{v i}$ : fuzzy rough advertisement cost per unit advertisement,

$\widetilde{\bar{h}}_{i}$ : fuzzy rough holding costs per unit item per unit time,

$\widetilde{\bar{s}}_{i}$ : fuzzy rough selling price per unit item.

3.2. Assumptions. For $i$ th $(i=1,2, \ldots, n)$ item, the following assumptions are made.

(i) Advertisements through different media like print media, electric media, and so forth are made to boost the demand.

(ii) Allowing shortages will have the adverse effect on the sale. Moreover, shortages always bring loss of goodwill to the firm. Hence, shortages are not allowed; that is, mathematically stock level is always greater than or equal to zero.

(iii) The promotional cost is introduced to provide the advertising that increases the demand of an item and it is taken as an exponential function.

(iv) The inflation rate $\left(k_{i}\right)$ and the discount rate $\left(r_{i}\right)$ representing the time value of money are crisp; therefore, the net discount rate of inflation is, $R_{i}=$ $r_{i}-k_{i}$.

(v) Unit production cost is produced-quantity dependent. This means that as some constant expenditure in production is spread over the number of produced units, unit production cost is inversely proportional to the produced quantity.

(vi) The inventory level, demand, and production are continuous variables with appropriate units.

(vii) There are $n$ items in the system.

(viii) The maximum space and investment are limited.

(ix) This is a single period inventory model with finite time horizon.

3.3. Model Formulation. A production-inventory system for $n$-items is considered with warehouse capacity and investment constraints. Here, the items are produced at a variable rate $U_{i}(t)$. Demand of the items is time dependent; it decreases due to the depreciation of sale and increases due to the advertising policy. So the promotional cost (cf. Datta et al. [5]) is introduced to provide the advertisement that increases the demand of an item and it is taken as a constant with zero promotional cost implying no advertise. The stock level at time $t$ decreases due to sale. Shortages are not allowed. The effect of inflation and time value of money are taken into consideration.

The differential equations for $i$ th item representing the above system during a fixed time-horizon $T$ are

$$
\begin{gathered}
\dot{X}_{i}(t)=\left(1-\delta_{i}\right) U_{i}(t)-D_{i}(t), \\
X_{i}(0)=0, \quad X_{i}(T)=X_{i T} .
\end{gathered}
$$

The demand rate is created by the advertisement and it is destroyed due to the depreciation of competition market, so the differential equation of demand for $i$ th item during the fixed time-horizon $T$ is

$$
\dot{D}_{i}(t)=V_{i}(t)-\beta_{i} D_{i}(t), \quad D_{i}(0)=D_{i 0}
$$

The net revenue from sale of fresh item and sale from the defective $i$ th item is

$$
\begin{aligned}
& \text { Maximize } J_{1}(U, V) \\
& =\sum_{i=1}^{n} \int_{0}^{T} e^{-R_{i} t}\left\{\widetilde{\bar{s}}_{i} D_{i}(t)+b_{\delta i} \delta_{i} U_{i}(t)\right\} d t+e^{-R_{i} T} b_{i} X_{i T} .
\end{aligned}
$$

Assuming the produced-quantity-dependent unit production cost, the warehouse of finite capacity and investment constraint, maximization of total profit consisting of sales proceeds, holding, promotional, and production costs lead to

$$
\begin{aligned}
& \text { Minimize } J_{2}(U, V) \\
& =\sum_{i=1}^{n} \int_{0}^{T} e^{-R_{i} t}\left\{\widetilde{\bar{h}}_{i} X_{i}(t)+\widetilde{\bar{C}}_{u i} U_{i}(t)+\widetilde{\bar{C}}_{v i} v_{i 0} e^{-\gamma_{i} t}\right\} d t
\end{aligned}
$$

subject to the constraints (11)-(12), and

$$
\begin{gathered}
\sum_{i=1}^{n} a_{i} X_{i}(t) \leq \widetilde{\widetilde{M}}, \quad 0 \leq t \leq T \text { (space constraint) } \\
\sum_{i=1}^{n} \int_{0}^{T}\left\{\widetilde{\bar{C}}_{u i} U_{i}(t)+\widetilde{\bar{C}}_{v i} v_{i 0} e^{-\gamma_{i} t}\right\} d t \\
\leq \widetilde{\bar{Z}} \text { (investment constraint) }
\end{gathered}
$$

3.3.1. Model with Linearly Time Dependent Advertisement Cost. In this case, we take the advertisement rate $V_{i}(t)=$ $v_{i 0} e^{-\gamma_{i} t}$ and the solving the equation (12); then, we get

$$
D_{i}(t)=D_{i}(0)\left(1-\beta_{i} t\right)+v_{i 0}\left\{t-\frac{\left(\gamma_{i}+\beta_{i}\right) t^{2}}{2}\right\} \text {. }
$$


From (11) and (12),

$$
\begin{aligned}
\dot{X}_{i}(t)= & \left(1-\delta_{i}\right)\left(u_{i 0}-u_{i 1} e^{-u_{i 2} t}\right) \\
& -D_{i}(0) e^{-\beta_{i} t}-v_{i 0}\left(\frac{e^{-\gamma_{i} t}-e^{-\beta_{i} t}}{\beta_{i}-\gamma_{i}}\right) .
\end{aligned}
$$

Integrating both sides and by mathematical approximation, we get

$$
\begin{aligned}
X_{i}(t)= & X_{i}(0)+\left(1-\delta_{i}\right)\left\{u_{i 0} t-\frac{u_{i 1}}{u_{i 2}}\left(1-e^{u_{i 2} t}\right)\right\} \\
& -\frac{D_{i}(0)}{\beta_{i}}\left(1-e^{-\beta_{i} t}\right)-\frac{v_{i 0}}{\beta_{i}-\gamma_{i}}\left(\frac{1-e^{-\gamma_{i} t}}{\gamma_{i} t}-\frac{1-e^{-\beta_{i} t}}{\beta_{i} t}\right) \\
X_{i}(t)= & X_{i}(0)+\left(1-\delta_{i}\right) \\
& \times\left\{u_{i 0} t-u_{i 1}\left(t-\frac{u_{i 2} t^{2}}{2}+\frac{u_{i 2}^{2} t^{3}}{6}-\cdots\right)\right\} \\
& -D_{i 0}\left(t-\frac{\beta_{i} t^{2}}{2}+\frac{\beta_{i}^{2} t^{3}}{6}-\cdots\right) \\
& -v_{i 0}\left\{\left(t-\frac{\gamma_{i} t^{2}}{2}+\frac{\gamma_{i}^{2} t^{3}}{6}-\cdots\right)\right. \\
& \left.+\cdots, \quad-\left(t-\frac{\beta_{i} t^{2}}{2}+\frac{\beta_{i}^{2} t^{3}}{6}-\cdots\right)\right\} \\
& +D_{i 0}\left(t-\frac{\beta_{i} T^{2}}{2}\right)-v_{i 0}\left\{\frac{T^{2}}{2}-\frac{\left(\beta_{i}+\gamma_{i}\right) T^{3}}{6}\right\} \\
& -D_{i 0}\left(t-\frac{\beta_{i} t^{2}}{2}\right)-v_{i 0}\left\{\frac{t^{2}}{2}-\frac{\left(\beta_{i}+\gamma_{i}\right) t^{3}}{6}\right\} \\
= & \left(1-\delta_{i}\right)\left\{u_{i 0} t-u_{i 1}\left(t-\frac{u_{i 2} t^{2}}{2}\right)\right\} \\
& \left.-\delta_{i}\right)\left\{u_{i 0} t-u_{i 1}\left(T-\frac{u_{i 2} T^{2}}{2}\right)\right\} \\
& +18) \\
& +\cdots
\end{aligned}
$$

Solving the equations (11) and (12), using (16), (18) and (19), we obtain the following equation (20) as:

Maximize $\widetilde{\bar{J}}_{1}(U, V)$

$$
\begin{aligned}
= & \sum_{i=1}^{n}\left\{\widetilde{\bar{s}}_{i} D_{i}(o)+b_{i} \delta_{i} u_{i 0}\right\} \frac{1-e^{-R_{i} T}}{R_{i}} \\
& +\left\{\widetilde{\bar{s}}_{i} v_{i 0}-\beta_{i}\right\}\left\{\frac{T e^{-R_{i} T}}{R_{i}}-\frac{1-e^{-R_{i} T}}{R_{i}^{2}}\right\}
\end{aligned}
$$

$$
\begin{aligned}
& -\widetilde{\bar{s}}_{i} v_{i 0} \frac{\left(\beta_{i}+\gamma_{i}\right) T^{3}}{6}-\frac{b_{\delta i} \delta_{i} u_{i 1}}{u_{i 2}+R_{i}}\left(1-e^{-\left(u_{i 2}+R_{i}\right) T}\right) \\
& +e^{-R_{i} T} b_{i} X_{i T}
\end{aligned}
$$

Minimize $\widetilde{\bar{J}}_{2}(U, V)$

$$
\begin{aligned}
&=\sum_{i=1}^{n} \widetilde{\bar{h}}_{i}[\left\{\left(1-\delta_{i}\right)\left(u_{i 0}-u_{i 1}\right)-D_{i}(0)\right\} \\
& \times\left\{\frac{1-e^{-R_{i} T}}{R_{i}^{2}}-\frac{T e^{-R_{i} T}}{R_{i}}\right\} \\
&+\left\{\left(1-\delta_{i}\right) u_{i 2}-D_{i}(0) \beta_{i}-v_{i 0}\right\} \\
& \times\left\{\frac{1-e^{-R_{i} T}}{R_{i}^{3}}-\frac{2 T e^{-R_{i} T}}{R_{i}^{2}}+\frac{T^{2} e^{-R_{i} T}}{R_{i}}\right\} \\
&-\left\{\frac{\left(\beta_{i}+\gamma_{i}\right) T}{6}\right\} \\
&\left.\times\left\{\frac{6\left(1-e^{-R_{i} T}\right)}{R_{i}^{4}}-\frac{\left(T^{2} R_{i}^{2}+3 T R_{i}+6\right) e^{-R_{i} T}}{R_{i}^{4}}\right\}\right] \\
&+\widetilde{\bar{C}}_{u i}\left\{\frac{u_{i 0}}{R_{i}}\left(1-e^{-R_{i} T}\right)-\frac{u_{i 1}}{u_{i 2}+R_{i}}\left(1-e^{-\left(u_{i 2}+R_{i}\right) T}\right)\right\} \\
& \widetilde{\bar{C}}_{v i} v_{i 0}\left(1-e^{-\left(\gamma_{i}+R_{i}\right) T}\right), \\
& \gamma_{i}+R_{i}
\end{aligned}
$$

(here $\simeq$ denotes the fuzzy rough of the parameters), where fuzzy rough variables $\widetilde{\bar{s}}_{i}, \widetilde{\bar{h}}_{i}, \widetilde{\bar{C}}_{u i}, \widetilde{\bar{C}}_{v i}, \widetilde{\bar{M}}, \widetilde{\bar{Z}}$ are defined as follows:

$$
\begin{aligned}
& \tilde{\bar{s}}_{i}=\left(\bar{s}_{i 1}, \bar{s}_{i 2}, \bar{s}_{i 3}, \bar{s}_{i 4}\right) \text { with } \bar{s}_{i t} \vdash\left(\left[s_{i t 2}, s_{i t 3}\right],\left[s_{i t 1}, s_{i t 4}\right]\right), 0 \leq \\
& s_{i t 1} \leq s_{i t 2}<s_{i t 3} \leq s_{i t 4}, \\
& \widetilde{\bar{h}}_{i}=\left(\bar{h}_{i 1}, \bar{h}_{i 2}, \bar{h}_{i 3}, \bar{h}_{i 4}\right) \text { with } \bar{h}_{i t} \vdash\left(\left[h_{i t 2}, h_{i t 3}\right],\left[h_{i t 1}, h_{i t 4}\right]\right), \\
& \quad 0 \leq h_{i t 1} \leq h_{i t 2}<h_{i t 3} \leq h_{i t 4}, \\
& \widetilde{\bar{c}}_{u i}=\left(\bar{c}_{u i 1}, \bar{c}_{u i 2}, \bar{c}_{i 3}, \bar{c}_{u i 4}\right) \text { with } \bar{c}_{u i t} \vdash\left(\left[c_{u i t 2}, c_{u i t 3}\right],\left[c_{u i t 1},\right.\right. \\
&\left.\left.c_{u i t 4}\right]\right), 0 \leq c_{u i t 1} \leq c_{u i t 2}<c_{u i t 3} \leq c_{u i t 4}, \\
& \widetilde{\bar{C}}_{v i}=\left(\bar{C}_{v i 1}, \bar{C}_{v i 2}, \bar{C}_{v i 3}, \bar{C}_{v i 4}\right) \text { with } \bar{C}_{v i t} \vdash\left(\left[C_{v i t 2}, C_{v i t 3}\right],\right. \\
&\left.\quad\left[C_{v i t 1}, C_{v i t 4}\right]\right), 0 \leq C_{v i t 1}<C_{v i t 2}<C_{v i t 3} \leq C_{v i t 4}, \\
& \widetilde{\bar{M}}=\left(\bar{M}_{1}, \bar{M}_{2}, \bar{M}_{3}, \bar{M}_{4}\right) \text { with } \bar{M}_{t} \vdash \quad\left(\left[M_{t 2}, M_{t 3}\right],\right. \\
&\left.\quad\left[M_{t 1}, M_{t 4}\right]\right), 0 \leq M_{t 1} \leq M_{t 2}<M_{t 3} \leq M_{t 4}, \\
& \widetilde{\bar{Z}}=\left(\bar{Z}_{1}, \bar{Z}_{2}, \bar{Z}_{3}, \bar{Z}_{4}\right) \text { with } \bar{Z}_{t} \vdash\left(\left[Z_{t 2}, Z_{t 3}\right],\left[Z_{t 1}, Z_{t 4}\right]\right), \\
& 0 \leq Z_{t 1} \leq Z_{t 2}<Z_{t 3} \leq Z_{t 4}, t=1,2,3,4 .
\end{aligned}
$$

\section{Solution of the Equivalent Crisp Inventory Model}

To solve the above fuzzy rough multiobjective multi-item production inventory model, we transform the above problem into its expected value model using Lemma 8 and 
Theorems 9 and 10. Thus, we have the following fuzzy rough expected value multiobjective inventory model:

$$
\begin{aligned}
& \max \left[E\left[\widetilde{J}_{1}(U, V)\right], E\left[-\widetilde{J}_{2}(U, V)\right]\right] \\
& \text { s.t. } \quad \sum_{i=1}^{n} a_{i}\left[\left(1-\delta_{i}\right)\left\{u_{i 0} t-u_{i 1}\left(t-\frac{u_{i 2} t^{2}}{2}\right)\right\}\right. \\
& -D_{i}(0)\left(t-\frac{\beta_{i} t^{2}}{2}\right) \\
& \left.-v_{i 0}\left\{\frac{t^{2}}{2}-\frac{\left(\beta_{i}+\gamma_{i}\right) t^{3}}{6}\right\}\right] \leq E[\widetilde{\bar{M}}] \\
& \sum_{i=1}^{n}\left[E [ \widetilde { C } _ { u i } ] \left\{\frac{u_{i 0}}{R_{i}}\left(1-e^{-R_{i} T}\right)\right.\right. \\
& \left.-\frac{u_{i 1}}{u_{i 2}+R_{i}}\left(1-e^{-\left(u_{i 2}+R_{i}\right) T}\right)\right\} \\
& \left.+\frac{E\left[\widetilde{\bar{C}}_{v i}\right] v_{i 0}}{\gamma_{i}+R_{i}}\left(1-e^{-\left(\gamma_{i}+R_{i}\right) T}\right)\right] \leq E[\widetilde{\bar{Z}}],
\end{aligned}
$$

where

$$
\begin{aligned}
& E\left[\widetilde{\bar{J}}_{1}(U, V)\right]=\sum_{i=1}^{n}\left\{E\left[\widetilde{\bar{s}}_{i}\right] D_{i}(o)+b_{i} \delta_{i} u_{i 0}\right\} \frac{1-e^{-R_{i} T}}{R_{i}} \\
& +\left\{E\left[\widetilde{\bar{s}}_{i}\right] v_{i 0}-\beta_{i}\right\}\left\{\frac{T e^{-R_{i} T}}{R_{i}}-\frac{1-e^{-R_{i} T}}{R_{i}^{2}}\right\} \\
& -E\left[\widetilde{\bar{s}}_{i}\right] v_{i 0} \frac{\left(\beta_{i}+\gamma_{i}\right) T^{3}}{6} \\
& -\frac{b_{\delta i} \delta_{i} u_{i 1}}{u_{i 2}+R_{i}}\left(1-e^{-\left(u_{i 2}+R_{i}\right) T}\right)+e^{-R_{i} T} b_{i} X_{i T}, \\
& E\left[\widetilde{\bar{J}}_{2}\right](U, V)=\sum_{i=1}^{n} E\left[\widetilde{\widetilde{h}}_{i}\right]\left[\left\{\left(1-\delta_{i}\right)\left(u_{i 0}-u_{i 1}\right)-D_{i}(0)\right\}\right. \\
& \times\left\{\frac{1-e^{-R_{i} T}}{R_{i}^{2}}-\frac{T e^{-R_{i} T}}{R_{i}}\right\} \\
& +\left\{\left(1-\delta_{i}\right) u_{i 2}-D_{i}(0) \beta_{i}-v_{i 0}\right\} \\
& \times\left\{\frac{1-e^{-R_{i} T}}{R_{i}^{3}}\right. \\
& \left.-\frac{2 T e^{-R_{i} T}}{R_{i}^{2}}+\frac{T^{2} e^{-R_{i} T}}{R_{i}}\right\} \\
& -\left\{\frac{\left(\beta_{i}+\gamma_{i}\right) T}{6}\right\}
\end{aligned}
$$

$$
\begin{gathered}
\times\left\{\frac{6\left(1-e^{-R_{i} T}\right)}{R_{i}^{4}}\right. \\
\left.\left.-\frac{\left(T^{2} R_{i}^{2}+3 T R_{i}+6\right) e^{-R_{i} T}}{R_{i}^{4}}\right\}\right] \\
+E\left[\widetilde{\bar{C}}_{u i}\right]\left\{\frac{u_{i 0}}{R_{i}}\left(1-e^{-R_{i} T}\right)\right. \\
\left.-\frac{u_{i 1}}{u_{i 2}+R_{i}}\left(1-e^{-\left(u_{i 2}+R_{i}\right) T}\right)\right\} \\
+\frac{E\left[\widetilde{\bar{C}}_{v i}\right] v_{i 0}}{\gamma_{i}+R_{i}}\left(1-e^{-\left(\gamma_{i}+R_{i}\right) T}\right),
\end{gathered}
$$

where

$$
\begin{array}{cc}
E\left[\widetilde{\bar{s}}_{i}\right]=\frac{1}{16} \sum_{t=1}^{4} \sum_{k=1}^{4} s_{i t k}, & E\left[\widetilde{\bar{h}}_{i}\right]=\frac{1}{16} \sum_{t=1}^{4} \sum_{k=1}^{4} h_{i t k}, \\
E\left[\widetilde{\bar{C}}_{u i}\right]=\frac{1}{16} \sum_{t=1}^{4} \sum_{k=1}^{4} C_{u i t k}, & E\left[\widetilde{\bar{C}}_{v i}\right]=\frac{1}{16} \sum_{t=1}^{4} \sum_{k=1}^{4} C_{v i t k} \\
E\left[\widetilde{\bar{M}}_{i}\right]=\frac{1}{16} \sum_{t=1}^{4} \sum_{k=1}^{4} M_{t k}, & E[\overline{\bar{Z}}]=\frac{1}{16} \sum_{t=1}^{4} \sum_{k=1}^{4} Z_{t k}, \\
\text { for } i & =1,2, \ldots, n .
\end{array}
$$

We also define the profit for the system $E(J)=E\left(J_{1}\right)=E\left(J_{2}\right)$.

4.1. Method 1: Solution by MOGA. The biobjectives given by (21) and (22) are directly solved by MOGA outlined in Section 2.4.

4.2. Method 2: Solution by GRG. To solve the multiobjective problem (21) by GRG technique, we use the following procedures as follows.

Step 1. Seek only the first objective function that is $E\left[\tilde{\bar{J}}_{1}(U, V)\right]$ and solve it as a single objective function by fuzzy rough simulation algorithm. The steps of fuzzy rough simulation algorithm are followed as per Liu [31].

Let $\left(U^{1}, V^{1}\right)$ be the optimal value of the decision variables and hence the optimal value of the objective function is $E\left[J_{1}\left(U^{1}, V^{1}\right)\right]$. Next, find the value of the second objective function $E\left[-J_{2}(U, V)\right]$ at $\left(U^{1}, V^{1}\right)$ that is equals to $E\left[-J_{2}\left(U^{1}, V^{1}\right)\right]$.

Step 2. In a similar way, pick up only the second objective function $E\left[-J_{2}(U, V)\right]$ and solve it by using fuzzy rough simulation algorithm. Let $\left(U^{2}, V^{2}\right)$ be the optimal value of the decision variables and hence the optimal value of the objective function is $E\left[-J_{2}\left(U^{2}, V^{2}\right)\right]$. Next, find the value of the first objective function $E\left[J_{1}(U, V)\right]$ at $\left(U^{2}, V^{2}\right)$ that is equals to $E\left[J_{1}\left(U^{2}, V^{2}\right)\right]$. 
Step 3. The objective functions will then be defined by the relations $E\left[J_{1}\left(U^{2}, V^{2}\right)\right] \leq E\left[J_{1}(U, V)\right] \leq E\left[J_{1}\left(U^{1}, V^{2}\right)\right]$ and $E\left[-J_{2}\left(U^{1}, V^{1}\right)\right] \leq E\left[-J_{2}(U, V)\right] \leq E\left[-J_{2}\left(U^{2}, V^{2}\right)\right]$.
Step 4. The membership functions corresponding to the objective functions of model (21) are then formulated as

$$
\begin{aligned}
& \mu_{E\left[\widetilde{J}_{1}\right]}(U, V)=\left\{\begin{array}{l}
1, \\
1-\frac{E\left[\widetilde{J}_{1}(U, V)\right]-E\left[\widetilde{J}_{1}\left(U^{2}, V^{2}\right)\right]}{E\left[\widetilde{J}_{1}\left(U^{1}, V^{1}\right)\right]-E\left[\widetilde{J}_{1}\left(U^{2}, V^{2}\right)\right]} \\
0,
\end{array}\right. \\
& \mu_{E\left[\widetilde{I}_{2}\right]}(U, V)=\left\{\begin{array}{l}
1, \\
1-\frac{E\left[-\widetilde{J}_{2}(U, V)\right]-E\left[-\widetilde{J}_{2}\left(U^{1}, V^{1}\right)\right]}{E\left[-\widetilde{J}_{2}\left(U^{2}, V^{2}\right)\right]-E\left[-\widetilde{J}_{2}\left(U^{1}, V^{1}\right)\right]}, \\
0,
\end{array}\right. \\
& \text { for } E\left[\widetilde{J}_{1}(U, V)\right] \geq E\left[\widetilde{J}_{1}\left(U^{1}, V^{1}\right)\right] \text {, } \\
& \text { for } E\left[\widetilde{J}_{1}\left(U^{2}, V^{2}\right)\right] \leq E\left[\widetilde{J}_{1}(U, V)\right] \\
& \leq E\left[\widetilde{J}_{1}\left(U^{1}, V^{1}\right)\right], \\
& \text { for } E\left[\widetilde{J}_{1}(U, V)\right]<E\left[\widetilde{J}_{1}\left(U^{2}, V^{2}\right)\right] \text {, } \\
& \text { for } E\left[-\widetilde{J}_{2}(U, V)\right] \geq E\left[-\widetilde{J}_{2}\left(U^{2}, V^{2}\right)\right] \text {, } \\
& \text { for } E\left[-\widetilde{J}_{2}\left(U^{1}, V^{1}\right)\right] \leq E\left[-\widetilde{J}_{2}(U, V)\right] \\
& <E\left[-\widetilde{J}_{2}\left(U^{2}, V^{2}\right)\right], \\
& \text { for } E\left[-\widetilde{J}_{2}(U, V)\right] \leq E\left[-\widetilde{J}_{2}\left(U^{1}, V^{1}\right)\right] \text {. }
\end{aligned}
$$

Step 5. Then, maximize the membership functions using a max-convex combination operator following Bellman and Zadeh [32]. Using weighted sum method for the membership functions $\mu_{E\left[\widetilde{J}_{1}\right]}(U, V)$ and $\mu_{E\left[\widetilde{J}_{2}\right]}(U, V)$, the multiobjective problem can be formulated in a single objective problem as

$$
\begin{aligned}
& \max \left[\omega_{1} \mu_{E\left[\widetilde{J}_{1}\right]}(U, V)+\omega_{2} \mu_{E\left[\widetilde{I}_{1}\right]}(U, V)\right] \\
& \text { s.t. } \quad \sum_{i=1}^{n} a_{i}\left[\left(1-\delta_{i}\right)\left\{u_{i 0} t-u_{i 1}\left(t-\frac{u_{i 2} t^{2}}{2}\right)\right\}\right. \\
& -D_{i}(0)\left(t-\frac{\beta_{i} t^{2}}{2}\right) \\
& \left.-v_{i 0}\left\{\frac{t^{2}}{2}-\frac{\left(\beta_{i}+\gamma_{i}\right) t^{3}}{6}\right\}\right] \leq E[\widetilde{M}] \\
& \sum_{i=1}^{n}\left[E [ \widetilde { \overline { C } } _ { u i } ] \left\{\frac{u_{i 0}}{R_{i}}\left(1-e^{-R_{i} T}\right)\right.\right. \\
& \left.-\frac{u_{i 1}}{u_{i 2}+R_{i}}\left(1-e^{-\left(u_{i 2}+R_{i}\right) T}\right)\right\} \\
& \left.+\frac{E\left[\widetilde{\bar{C}}_{v i}\right] v_{i 0}}{\gamma_{i}+R_{i}}\left(1-e^{-\left(\gamma_{i}+R_{i}\right) T}\right)\right] \leq E[\overline{\bar{Z}}],
\end{aligned}
$$

(where $\omega_{1}+\omega_{2}=1, \omega_{1}, \omega_{2} \geq 0$ ). Then, the problem (25) now can be solved by the GRG technique (Lingo-11.0).

\section{Numerical Experiment}

5.1. Collected of Data from a Firm. With the development and innovation of technology, Remond Collon Mill company,
Kolkata, India, produces two new products. The production cost, holding cost, selling price, and others are considered as trapezoidal fuzzy rough variables. The advertisement cost is also trapezoidal fuzzy rough variables. The defective products are sold by a reduced price. At a particular time of each year, the company offers the reduction sale to finish the stock. The collected data are given in Tables 1 and 2. In this case, we take two items, that is, $n=2$ and the business period $T=5$ months.

5.2. Optimal Results. For the above data, we optimized (21) and (22) using MOGA with the parameters, POPSIZE $=50$ and $\mathrm{PCROS}=0.2, \mathrm{PMUTE}=0.2, \mathrm{MAXGEN}=50 . \mathrm{A}$ real-number presentation is used here. In this representation, each chromosome $X$ is a string of $n$ number of genes where these $n$ numbers of genes, respectively, represent $n$ (here, $n=4)$ number of decision variables $X_{i}, U_{i}, D_{i}, i=1,2$. The individual optimum values of the objective functions are $E\left[J_{1}^{*}\right]=65.156 \$$ and $E\left[J_{2}^{*}\right]=56.279 \$$, and using (6)-(7), from (18), we get the optimal revenue, rates of advertisement, and production functions as $J=983.5 \$, V_{1}=0.4 t, V_{2}=$ $0.3 t, U_{1}(t)=27.4+1.3 t, 0 \leq t \leq 3.5$, and $U_{2}(t)=$ $22.5+0.5 t, 0 \leq t \leq 3.6$. We obtain the optimum values of $X_{i}(t), U_{i}(t), V_{i}(t)$ and $D_{i}(t)(i=1,2)$ and present in Table 3. The graphical changes are also depicted in Figure 2 for first item. Optimum results of the objective function (25) are obtained by GRG method for different values of $w_{1}$ and $w_{2}$. The results obtained by GRG and MOGA are presented in Table 4.

5.3. Sensitivity Analysis. Sensitivity analysis is made for linearly time dependent advertisement cost to study the effect of changing the holding cost and selling price $h_{i}$ and $s_{i}$, $i=1,2$ on the objective value. Percentage changes $h_{i}, s_{i}$ of these values are shown with respect to the values used in the original problem in Table 5 . If $h_{1}, h_{2}$ are changed by $+10 \%$, $-10 \%,+15 \%,-15 \%,+20 \%$, and $-20 \%$, the corresponding 
TABLE 1: The collected crisp data.

\begin{tabular}{lcccccccc}
\hline Item & $D_{i}(0)$ & $\beta_{i}$ & $b_{i}$ & $b_{\delta i}$ & $a_{i}$ & $r_{i}$ & $k_{i}$ \\
\hline 1 & 50 & 0.03 & 1399 & 899 & 1.5 & 0.079 & 1.45 \\
2 & 40 & 0.02 & 1299 & 799 & 1.6 & 0.081 & 1.51 & 0.1 \\
\hline
\end{tabular}

TABLE 2: The collected data for fuzzy rough variables.

\begin{tabular}{|c|c|c|c|}
\hline Item & $\widetilde{\bar{C}}_{u i}$ & $\tilde{\bar{h}}_{i}$ & $\tilde{\bar{s}}_{i}$ \\
\hline \multirow{2}{*}{1} & $\left(\zeta_{1}-20, \zeta_{1}-10, \zeta_{1}+10, \zeta_{1}+20\right)$ & $\left(\gamma_{1}-3, \gamma_{1}-2, \gamma_{1}+2, \gamma_{1}+3\right)$ & $\left(v_{1}-5, v_{1}-3, v_{1}+3, v_{1}+5\right)$ \\
\hline & $\zeta_{1}=([700,820],[600,910])$ & $\gamma_{1}=([27,30],[24,37])$ & $v_{1}=([1400,1645],[1325,1840])$ \\
\hline \multirow{2}{*}{2} & $\left(\zeta_{2}-20, \zeta_{2}-10, \zeta_{2}+10, \zeta_{1}+20\right)$ & $\left(\gamma_{2}-3, \gamma_{2}-2, \gamma_{2}+2, \gamma_{2}+3\right)$ & $\left(v_{2}-5, v_{2}-3, v_{2}+3, v_{2}+5\right)$ \\
\hline & $\zeta_{2}=([650,790],[600,960])$ & $\gamma_{2}=([26,29],[23,39])$ & $\nu_{2}=([1325,1600],[1210,1860])$ \\
\hline Item & $\widetilde{\bar{C}}_{v i}$ & $\widetilde{\bar{M}}$ & $\widetilde{\bar{Z}}$ \\
\hline \multirow[t]{2}{*}{1} & $\left(\rho_{1}-2, \rho_{1}-1, \rho_{1}+1, \rho_{1}+2\right)$ & $\left(\tau_{1}-8, \tau_{1}-9, \tau_{1}+8, \tau_{1}+9\right)$ & $\left(\chi_{1}-9, \chi_{1}-7, \chi_{1}+7, \chi_{1}+9\right)$ \\
\hline & $\rho_{1}=([75,150],[50,340])$ & $\tau_{1}=([1290,1310],[1260,1350])$ & $\chi_{1}=([800000,910000],[710000,950000])$ \\
\hline \multirow{2}{*}{2} & $\left(\rho_{2}-2, \rho_{2}-1, \rho_{2}+1, \rho_{2}+2\right)$ & $\left(\tau_{2}-9, \tau_{2}-8 \tau_{2}+8, \tau_{2}+9\right)$ & $\left(\chi_{2}-9, \chi_{2}-7, \chi_{2}+9, \chi_{2}+7\right)$ \\
\hline & $\rho_{2}=([70,145],[45,300])$ & $\tau_{2}=([1295,1325],[1280,1348])$ & $\chi_{2}=([750000,850000],[700000,950000])$ \\
\hline
\end{tabular}

TABLE 3: Optimum values of $X_{i}(t), U_{i}(t)$, and $D_{i}(t)$ via MOGA.

\begin{tabular}{lccccccc}
\hline$t$ & item & 0 & 1 & 2 & 3 & 4 & \\
\hline \multirow{2}{*}{$X_{i}(t)$} & 1 & 0 & 7.1 & 10.8 & 12.4 & 7 & 0 \\
& 2 & 0 & 3.2 & 5 & 3.2 & 4.2 & 0 \\
\multirow{2}{*}{$U_{i}(t)$} & 1 & 27.4 & 28.7 & 23.0 & 31.30 & 0 & 0 \\
& 2 & 22.5 & 23.0 & 24.00 & 24.60 & 25.25 \\
$D_{i}(t)$ & 1 & 25.0 & 23.0 & 19.80 & 20.15 & 20.80 \\
& 2 & 20.0 & 19.7 & & & 21.73 \\
\hline
\end{tabular}

TABLE 4: Results obtained are compared via GRG and MOGA.

\begin{tabular}{lcccc}
\hline Change of $w_{i}$ & Process & $E\left[J_{1}^{*}\right]$ & $E\left[J_{2}^{*}\right]$ & 57.13 \\
\hline$w_{1}=0.5, w_{2}=0.5$ & GRG & 64.44 & 56.28 & 8.17 \\
& MOGA & 65.13 & 571.13 & 8.574 \\
$w_{1}=0.7, w_{2}=0.3$ & GRG & 64.44 & 56.28 & 8.173 \\
& MOGA & 65.13 & 57.13 & 8.57 \\
$w_{1}=0.3, w_{2}=0.7$ & GRG & 64.44 & 56.28 & 8.17 \\
\hline
\end{tabular}

TABLE 5: Values of the objective function, $E\left[J^{*}\right]$ for different values of $h_{i}, s_{i}, i=1,2$.

\begin{tabular}{lccc}
\hline Changes in $h_{i}$ & Changes in $E\left[J^{*}\right]$ due to & $\begin{array}{c}\text { Changes in } s_{i} \text { due to } \\
h_{i}=([1400,1650],[1325,1840])\end{array}$ & $\begin{array}{c}\text { Changes in } E\left[J^{*}\right] \text { due to } \\
h_{i}=([27,30],[24,37])\end{array}$ \\
\hline $10 \%$ & $-0.37 \%$ & $4.78 \%$ & $-0.21 \%$ \\
$15 \%$ & $-0.87 \%$ & $5.36 \%$ & $-0.27 \%$ \\
$20 \%$ & $-0.97 \%$ & $6.17 \%$ & $-0.14 \%$ \\
$-10 \%$ & $0.47 \%$ & $-3.51 \%$ & $0.19 \%$ \\
$-15 \%$ & $0.78 \%$ & $-4.73 \%$ & $0.67 \%$ \\
$-20 \%$ & $0.98 \%$ & $-8.64 \%$ & $0.78 \%$ \\
\hline
\end{tabular}

changes in values of the objective function are $-0.37 \%$, $+0.47 \%,-0.87 \%,+0.98 \%,-0.97 \%$, and $+0.99 \%$, respectively, and if $s_{1}, s_{2}$ are increased/reduced by $+10 \%,-10 \%,+15 \%$, $-15 \%,+20 \%$, and $-20 \%$, the values of the objective function are changed by $+4.78 \%,-3.51 \%,+5.36 \%,-4.73 \%,+6.18 \%$, and $-8.68 \%$. From these results, we see that the value of the objective function is more sensitive to the changes in the inventory holding cost $h_{i}, i=1,2$. The value of the objective function is highly sensitively to the changes in the unit selling price $s_{i}, i=1,2$. 


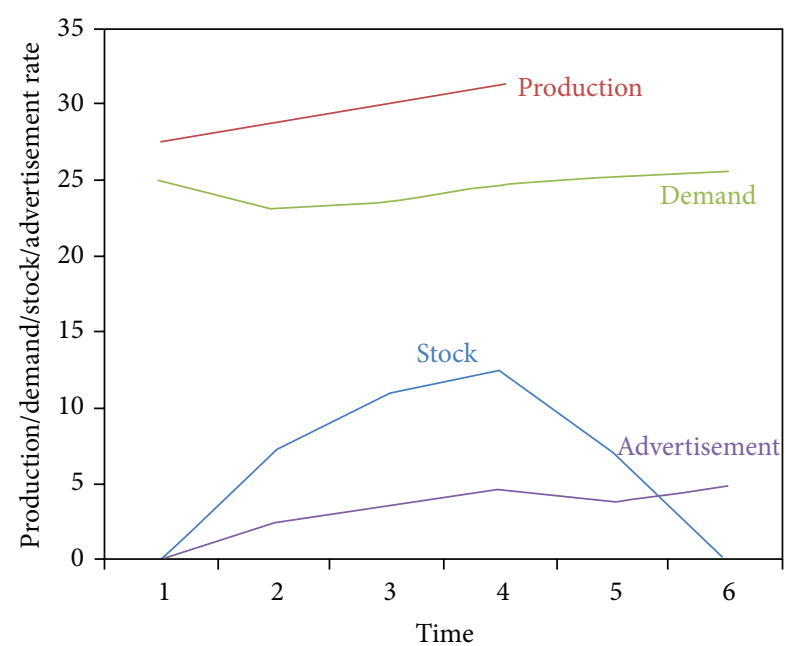

FIGURE 2: Optimal production, stock and demand for Fu-Ro advertisement rate.

\section{Conclusion}

The present paper deals with the optimum production and advertising policy for a multi-item multiobjective production inventory system with depreciation rate of sales, salvage value, space capacity constraint, investment constraint, and advertisement control demand under inflation and time discounting environment in Fu-Ro environment. Also some ideas such as (i) optimal control production problem for defective multi-items, (ii) advertisement dependent demand, (iii) fuzzy rough inventory costs, and (iv) inflation and imprecise depreciation of money values have been introduced for the first time. In the solution approach, the new idea of expectation for fuzzy rough variable to get their corresponding crisp values is used. In this connection, Lemma 8 and Theorems 9 and 10 have been developed for the first time. Analysis presented here can be extended to other productioninventory problems with different types of demand, advertisement, deterioration, defectiveness, price discount, and so forth.

\section{Conflict of Interests}

The authors declare that there is no conflict of interests regarding the publication of this paper.

\section{References}

[1] J. A. Buzacott, "Economic order quantities with inflation," Operational Research Quarterly, vol. 26, no. 3, pp. 553-558, 1975.

[2] R. B. Misra, "A note on optimal inventory management under inflation," Naval Research Logistics, vol. 26, no. 1, pp. 161-165, 1979.

[3] S. Lo, H. Wee, and W. Huang, "An integrated productioninventory model with imperfect production processes and Weibull distribution deterioration under inflation," International Journal of Production Economics, vol. 106, no. 1, pp. 248260, 2007.
[4] D. I. Cho, "Analysis of optimal production and advertising policies," International Journal of Systems Science, vol. 27, no. 12, pp. 1297-1305, 1996.

[5] T. K. Datta, K. Paul, and A. K. Pal, "Demand promotion by upgradation under stock-dependent demand situation: a model," International Journal of Production Economics, vol. 55, no. 1, pp. 31-38, 1998.

[6] C. K. Sivashankari and S. Panayappan, "Production inventory model with reworking of imperfect production, scrap and shortages," International Journal of Management Science and Engineering Management, vol. 9, no. 1, pp. 9-20, 2013.

[7] C. Krishnamoorthi and S. Panayappan, "An EPQ model for imperfect production systems with rework and incorporating a multi-delivery policy," International Journal of Management Science and Engineering Management, vol. 8, no. 2, pp. 142-152, 2013.

[8] S. Mandal and D. A. Khan, "Theory and methodology for the JELS model in one-vendor, multi-customer situations when costs are fuzzy and lot sizes deterministic," International Journal of Management Science and Engineering Management, vol. 8, no. 3, pp. 210-218, 2013.

[9] L. A. Zadeh, "Fuzzy sets," Information and Computation, vol. 8, pp. 338-353, 1965.

[10] L. A. Zadeh, "Fuzzy sets as a basis for a theory of possibility," Fuzzy Sets and Systems, vol. 1, no. 1, pp. 3-28, 1978.

[11] D. Dubois and H. Prade, Possibility Theory: An Approach to Computerized Processing of Uncertainty, Plenum Press, New York, NY, USA, 1988.

[12] D. K. Jana, K. Maity, and T. K. Roy, "A three-layer supply chain integrated production-inventory model under permissible delay in payments in uncertain environments," Journal of Uncertainty Analysis and Applications, vol. 1, no. 1, pp. 1-17, 2013.

[13] D. Dubois and H. Prade, " Twofold fuzzy sets and rough setssome issues in knowledge representation," Fuzzy Sets and Systems, vol. 23, no. 1, pp. 3-18, 1987.

[14] D. Dubois and H. Prade, "Rough fuzzy sets and fuzzy rough sets," Fuzzy Sets and Systems, vol. 17, pp. 191-208, 1990.

[15] N. N. Morsi and M. M. Yakout, "Axiomatics for fuzzy rough sets," Fuzzy Sets and Systems, vol. 100, pp. 327-342, 1998.

[16] J. Xu and L. Zhao, "A class of fuzzy rough expected value multiobjective decision making model and its application to inventory problems," Computers \& Mathematics with Applications, vol. 56, no. 8, pp. 2107-2119, 2008.

[17] Z. Tao and J. Xu, "A class of rough multiple objective programming and its application to solid transportation problem," Information Sciences, vol. 188, pp. 215-235, 2012.

[18] K. Maity and M. Maiti, "Numerical approach of multi-objective optimal control problem in imprecise environment," Fuzzy Optimization and Decision Making, vol. 4, no. 4, pp. 313-330, 2005.

[19] J. Xu and L. Zhao, "A multi-objective decision-making model with fuzzy rough coefficients and its application to the inventory problem," Information Sciences, vol. 180, no. 5, pp. 679-696, 2010.

[20] J. H. Holland, Adaptation in Natural and Artificial Systems, University of Michigan, Ann Arbor, Mich, USA, 1975.

[21] C. M. Fonseca and P. J. Fleming, "Genetic algorithm for multiobjective optimization, formulation, discussion and generalization," in Proceedings of the 5th International Conference on Genetic Algorithm, S. Forrest, Ed., pp. 416-423, Morgan Kauffman, San Mateo, Calif, USA, 1993. 
[22] N. Srinivas and K. Deb, "Multiobjective function optimization using nondominated sorting genetic algorithms," Evoluation Computation, vol. 2, pp. 221-248, 1995.

[23] K. Deb, A. Pratap, S. Agarwal, and T. Meyarivan, "A fast and elitist multiobjective genetic algorithm: NSGA-II," IEEE Transactions on Evolutionary Computation, vol. 6, no. 2, pp. 182197, 2002.

[24] D. K. Jana, K. Maity, B. Das, and T. K. Roy, "A fuzzy simulation via contractive mapping genetic algorithm approach to an imprecise production inventory model under volume flexibility," Journal of Simulation, vol. 7, no. 2, pp. 90-100, 2013.

[25] H. Lee and J. Yao, "Economic production quantity for fuzzy demand quantity and fuzzy production quantity," European Journal of Operational Research, vol. 109, no. 1, pp. 203-211, 1998.

[26] A. A. Taleizadeh, S. J. Sadjadi, and S. T. A. Niaki, "Multiproduct EPQ model with single machine, backordering and immediate rework process," European Journal of Industrial Engineering, vol. 5, no. 4, pp. 388-411, 2011.

[27] Z. T. Balkhi and A. Foul, "A multi-item production lot size inventory model with cycle dependent parameters," International Journal of Mathematical Models and Methods in Applied Sciences, vol. 3, no. 2, pp. 94-104, 2009.

[28] G. A. Gabriele and K. M. Ragsdell, "The generalized reduced gradient method," AMSE Journal of Engineering for Industry, vol. 99, no. 2, pp. 394-400, 1977.

[29] J. Xu and X. Zhou, Fuzzy Link Multiple-Objective Decision Making, Springer, Berlin,Germany, 2009.

[30] D. K. Jana, K. Maity, and T. K. Roy, "Multi-objective imperfect production inventory model in fuzzy rough environment via genetic algorithm," International Journal of Operational Research, vol. 18, no. 4, pp. 365-385, 2013.

[31] B. Liu, Theory and Practice of Uncertain Programming, Physica, Heidelberg, Germany, 2002.

[32] R. E. Bellman and L. A. Zadeh, "Decision-making in a fuzzy environment," Management Science, vol. 17, pp. B141-B164, 1970. 


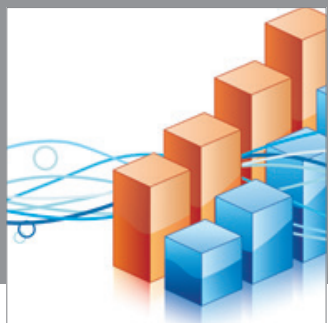

Advances in

Operations Research

mansans

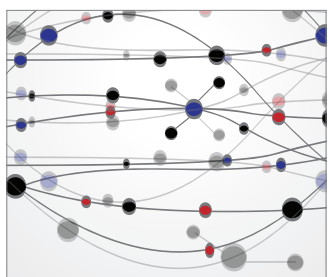

The Scientific World Journal
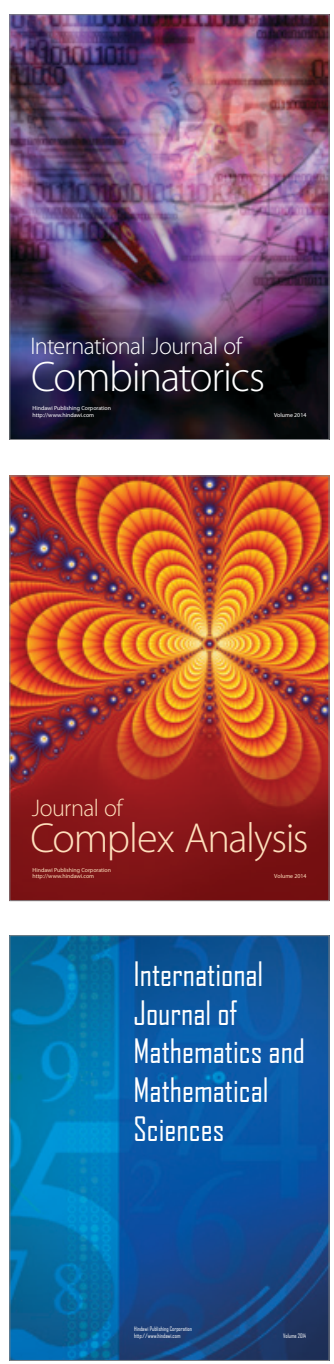
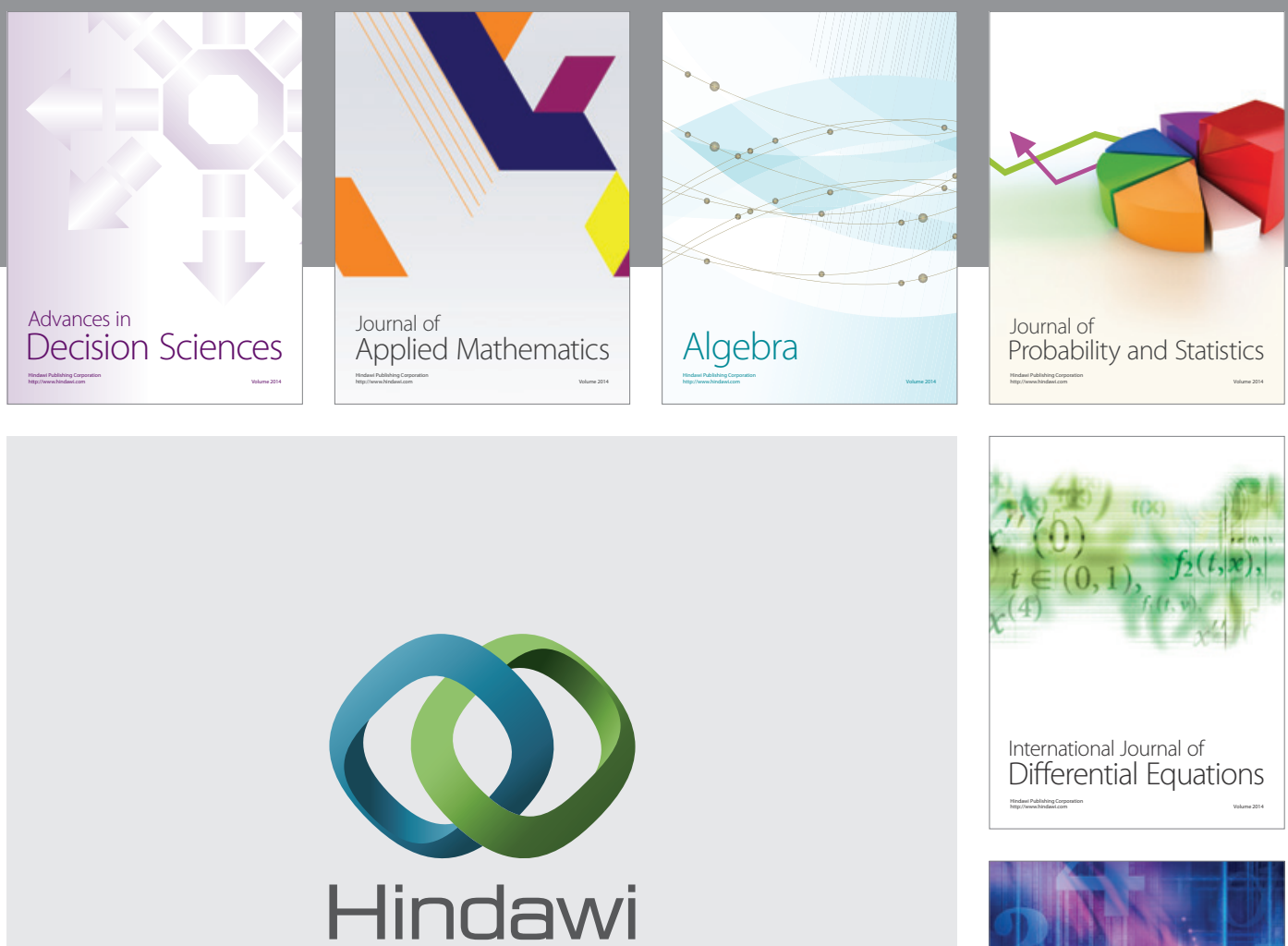

Submit your manuscripts at http://www.hindawi.com
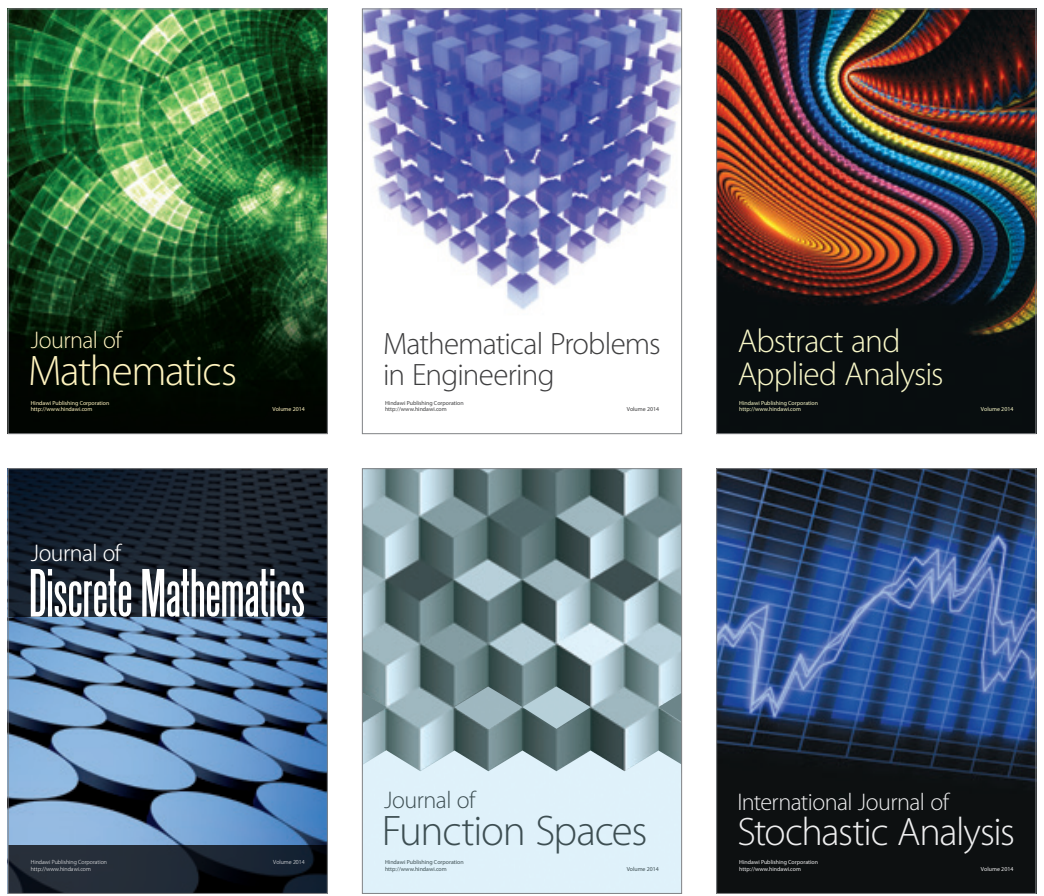

Journal of

Function Spaces

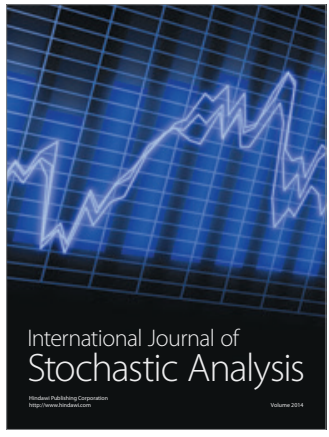

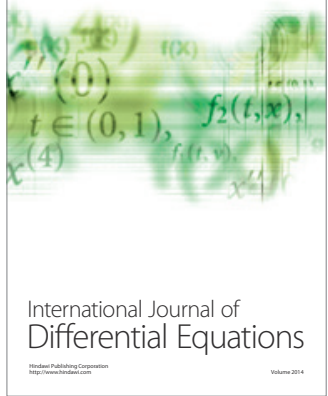
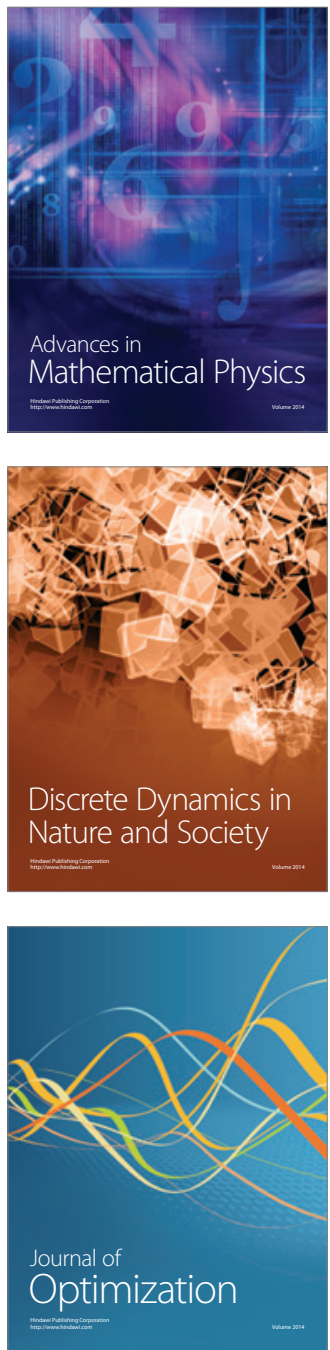